\title{
ON THE HERMITE-HADAMARD-MERCER TYPE INEQUALITIES FOR GENERALIZED PROPORTIONAL FRACTIONAL INTEGRALS
}

\author{
SEDA KILINÇ YILDIRIM AND HÜSEYIN YILDIRIM
}

\begin{abstract}
Our aim in this paper is to establish some new Hermite-HadamardMercer type integral inequalities by utilizing the fractional proportional-integral operators.For this purpose, Hermite-Hadamard-Mercer inequalities for differantiable mappings whose derivatives in absolute value are convex.
\end{abstract}

\section{INTRODUCTION AND PRELIMINARES}

Over the past few years, various researchers studied the so-called conformable integrals and derivatives. According to this idea, some authors used modified proportional derivatives to create nonlocal fractional integrals and derivatives, called fractional proportional integrals and derivatives, including see exponential functions in their kernels ([5], [7], [31], [32], [33]). Our purpose here new Hermite-HadamardMercer integral inequalities in the article some convex functions using fractional proportional integral operators.

Let $0<x_{1} \leq x_{2} \leq \cdots \leq x_{n}, \lambda=\left(\lambda_{1}, \lambda_{2}, \cdots, \lambda_{n}\right)$ nonnegative and $\sum_{j=1}^{n} \lambda_{j}=1$. The Jensen inequality [13] in literature states that if $f$ is a convex function then,

$$
f\left(\sum_{j=1}^{n} \lambda_{j} x_{j}\right) \leq \sum_{j=1}^{n} \lambda_{j} f\left(x_{j}\right) .
$$

The $f: J \subset \mathbb{R} \rightarrow \mathbb{R}$ be a convex function defined on an interval $J$ of real numbers $\theta, \beta \in J$ and $\theta<\beta$, if the following inequalities

$$
f\left(\frac{\theta+\beta}{2}\right) \leq \frac{1}{\beta-\theta} \int_{\theta}^{\beta} f(x) d x \leq \frac{f(\theta)+f(\beta)}{2},
$$

holds, it is called Hermite-Hadamard type inequality [5].

Theorem 1. [21] Let $f$ is a convex function on $[\theta, \beta]$, then

$$
f\left(\theta+\beta-\sum_{j=1}^{n} \lambda_{j} x_{j}\right) \leq f(\theta)+f(\beta)-\sum_{j=1}^{n} \lambda_{j} f\left(x_{j}\right)
$$

for each $x_{j} \in[\theta, \beta]$ and $\lambda_{j} \in[0,1](j=1,2, \cdots, n)$ with $\sum_{j=1}^{n} \lambda_{j}=1$.

Jensen-Mercer inequality, see ([1], [18], [20], [23] .)

\footnotetext{
Key words and phrases. Convex function, Hermite Hadamard inequalities, Jensen Inequalities, Jensen-Mercer İnequality, Fractional integrals, Fractional proportional integrals,Fractional integral inequalities.
}

2010 Mathematics Subject Classification. 26A33, 26A51, 26D15, 26D10, 26D53. 
Definition 1. [7] Let $f \in L[\theta, \beta]$. The Generalized proportional integral operators $J_{\theta^{+}}^{\beta, \xi}$ fand $J_{\beta^{-}}^{\beta, \xi}$ of order are defined by

$$
\left(J_{\theta^{+}}^{\beta, \xi} f\right)(x)=\frac{1}{\xi^{\beta} \Gamma(\beta)} \int_{\theta}^{x} \exp \left[\frac{\xi-1}{\xi}(x-\tau)\right](x-\tau)^{\beta-1} f(\tau) d \tau, \quad x>\theta
$$

and

$$
\left(J_{\beta^{-}}^{\beta, \xi} f\right)(x)=\frac{1}{\xi^{\beta} \Gamma(\beta)} \int_{x}^{\beta} \exp \left[\frac{\xi-1}{\xi}(\tau-x)\right](\tau-x)^{\beta-1} f(\tau) d \tau, \quad \beta>x .
$$

where $\xi \in(0,1]$ and $\beta \in \mathbb{C}$ and $\Re(\beta)>0$ and $\Gamma$ is known gamma function. For more details may consult see ([7], [29], [32].)

If we consider $\xi=1$ in (1.3) and (1.4) in classical Riemann-Liouville fractional integrals are obtained.

\section{Main Results}

Using the Jensen-Mercer inequality, Hermite Hadamard inequalities can be represented in generalized proportional fractional integral forms as follows.

Theorem 2. Let $f$ be a positive continuous, decreasing and convex function on the interval $[\theta, \beta]$, then the following inequality for, generalized proportional fractional integrals holds;

$$
\begin{aligned}
& \frac{1}{\xi^{\sigma}}\left[\frac{1}{\sigma}+\sum_{n=1}^{\infty}\left(\frac{\xi-1}{\xi}\right)^{n} \frac{1}{\sigma+n}\right] f\left(\theta+\beta-\frac{x+y}{2}\right) \\
& \leq \frac{1}{\xi^{\sigma}}\left[\frac{1}{\sigma}+\sum_{n=1}^{\infty}\left(\frac{\xi-1}{\xi}\right)^{n} \frac{1}{\sigma+n}\right][f(\theta)+f(\beta)] \\
& -\frac{\Gamma(\sigma)}{2(y-x)^{\sigma}}\left[J_{x+\xi}^{\sigma, \xi} f(y)+J_{y^{-}}^{\sigma, \xi} f(x)\right] \\
& \leq \frac{1}{\xi^{\sigma}}\left[\frac{1}{\sigma}+\sum_{n=1}^{\infty}\left(\frac{\xi-1}{\xi}\right)^{n} \frac{1}{\sigma+n}\right]\left[f(\theta)+f(\beta)-f\left(\frac{x+y}{2}\right)\right]
\end{aligned}
$$

and

$$
\begin{aligned}
& \frac{1}{\xi^{\sigma}}\left[\frac{1}{\sigma}+\sum_{n=1}^{\infty}\left(\frac{\xi-1}{\xi}\right)^{n} \frac{1}{\sigma+n}\right] f\left(\theta+\beta-\frac{x+y}{2}\right) \\
& \leq \frac{\Gamma(\sigma)}{2(y-x)^{\sigma}}\left[J_{(\theta+\beta-y)^{+}}^{\sigma, \xi} f(\theta+\beta-x)+J_{(\theta+\beta-x)^{-}}^{\sigma, \xi} f(\theta+\beta-y)\right] \\
& \leq \frac{1}{\xi^{\sigma}}\left[\frac{1}{\sigma}+\sum_{n=1}^{\infty}\left(\frac{\xi-1}{\xi}\right)^{n} \frac{1}{\sigma+n}\right]\left[\frac{f(\theta+\beta-x)+f(\theta+\beta-y)}{2}\right] \\
& \leq \frac{1}{\xi^{\sigma}}\left[\frac{1}{\sigma}+\sum_{n=1}^{\infty}\left(\frac{\xi-1}{\xi}\right)^{n} \frac{1}{\sigma+n}\right]\left[f(\theta)+f(\beta)-\frac{f(x)+f(y)}{2}\right]
\end{aligned}
$$

Then where $\xi \in(0,1]$ for all $x, y \in[\theta, \beta], \sigma \in \mathbb{C}$ and $\Re(\sigma)>0$.

Proof. Using the Jensen -Mercer inequality, we have

$$
f\left(\theta+\beta-\frac{x_{1}+y_{1}}{2}\right) \leq f(\theta)+f(\beta)-\frac{f\left(x_{1}\right)+f\left(y_{1}\right)}{2}
$$

for all $x_{1}, y_{1} \in[\theta, \beta]$. By changing of the variable $x_{1}=\tau x+(1-\tau) y$ and $y_{1}=$ $(1-\tau) x+\tau y, \quad x, y \in[\theta, \beta]$ and $\tau \in[0,1]$ in $(2.3)$, we obtain

$$
f\left(\theta+\beta-\frac{x+y}{2}\right) \leq f(\theta)+f(\beta)-\frac{f(\tau x+(1-\tau) y)+f((1-\tau) x+\tau y)}{2}
$$


Multiplying both sides of (2.4) by $\frac{\tau^{\sigma-1} e^{\frac{\xi-1}{\xi} \tau}}{\xi^{\sigma}}$ and integrate with respect to $\tau$ over $[0,1]$, we get

$$
\begin{aligned}
& \int_{0}^{1} \frac{\tau^{\sigma-1} e^{\frac{\xi-1}{\xi} \tau}}{\xi^{\sigma}} f\left(\theta+\beta-\frac{x+y}{2}\right) d \tau \leq[f(\theta)+f(\beta)] \int_{0}^{1} \frac{\tau^{\sigma-1} e^{\frac{\xi-1}{\xi} \tau}}{\xi^{\sigma}} d \tau \\
& -\int_{0}^{1} \frac{\tau^{\sigma-1} e^{\frac{\xi-1}{\xi}} \tau}{\xi^{\sigma}}\left[\frac{f(\tau x+(1-\tau) y)+f((1-\tau) x+\tau y)}{2}\right] d \tau \\
& \frac{1}{\xi^{\sigma}}\left[\frac{1}{\sigma}+\sum_{n=1}^{\infty}\left(\frac{\xi-1}{\xi}\right)^{n} \frac{1}{\sigma+n}\right] f\left(\theta+\beta-\frac{x+y}{2}\right) \\
& \leq\left[\frac{1}{\sigma}+\sum_{n=1}^{\infty}\left(\frac{\xi-1}{\xi}\right)^{n} \frac{1}{\sigma+n}\right][f(\theta)+f(\beta)] \\
& -\frac{1}{2} \int_{0}^{1} \frac{\tau^{\sigma-1} e^{\frac{\xi-1}{\xi} \tau}}{\xi^{\sigma}} f(\tau x+(1-\tau) y) d \tau \\
& -\frac{1}{2} \int_{0}^{1} \frac{\tau^{\sigma-1} e^{\frac{\xi-1}{\xi} \tau}}{\xi^{\sigma}} f((1-\tau) x+\tau y) d \tau
\end{aligned}
$$

i.e

$$
\begin{aligned}
& \frac{1}{\xi^{\sigma}}\left[\frac{1}{\sigma}+\sum_{n=1}^{\infty}\left(\frac{\xi-1}{\xi}\right)^{n} \frac{1}{\sigma+n}\right] f\left(\theta+\beta-\frac{x+y}{2}\right) \\
& \leq\left[\frac{1}{\sigma}+\sum_{n=1}^{\infty}\left(\frac{\xi-1}{\xi}\right)^{n} \frac{1}{\sigma+n}\right][f(\theta)+f(\beta)] \\
& -\frac{\Gamma(\sigma)}{2(y-x)^{\sigma}}\left[J_{x^{+}}^{\sigma, \xi} f(y)+J_{y^{-}}^{\sigma, \xi} f(x)\right] .
\end{aligned}
$$

and so the first inequality of (2.1) proved. To be able to prove the second inequality in (2.1) , first we have to pay attention to that if $f$ is a convex function, in case that, for $\tau \in[0,1]$, it gives

$$
\begin{aligned}
& f\left(\frac{x+y}{2}\right)=f\left(\frac{\tau x+(1-\tau) y+(1-\tau) x+\tau y}{2}\right) \\
& \leq \frac{f(\tau x+(1-\tau) y)+f((1-\tau) x+\tau y)}{2}
\end{aligned}
$$

Multiplying both sides of (2.8) by $\frac{\tau^{\sigma-1} e^{\frac{\xi-1}{\xi} \tau}}{\xi^{\sigma}}$ and integrate with respect to $\tau$ over $[0,1]$, we have

$$
\begin{aligned}
& \frac{1}{\xi^{\sigma}}\left[\frac{1}{\sigma}+\sum_{n=1}^{\infty}\left(\frac{\xi-1}{\xi}\right)^{n} \frac{1}{\sigma+n}\right] f\left(\frac{x+y}{2}\right) \\
\leq & \frac{1}{2} \int_{0}^{1} \frac{\tau^{\sigma-1} e^{\frac{\xi-1}{\xi} \tau}}{\xi^{\sigma}} f(\tau x+(1-\tau) y) d \tau \\
+ & \frac{1}{2} \int_{0}^{1} \frac{\tau^{\sigma-1} e^{\frac{\xi-1}{\xi} \tau}}{\xi^{\sigma}} f((1-\tau) x+\tau y) d \tau \\
= & \frac{\Gamma(\sigma)}{2(y-x)^{\sigma}}\left[J_{x^{+}}^{\sigma, \xi} f(y)+J_{y^{-}}^{\sigma, \xi} f(x)\right] .
\end{aligned}
$$

Adding $[f(\theta)+f(\beta)] \int_{0}^{1} \frac{\tau^{\sigma-1} e^{\frac{\xi-1}{\xi} \tau}}{\xi^{\sigma}}$ to both sides of (2.7) we find the second inequality of $(2.1)$.

now we prove the inequality $(2.2)$. From the convexity of $f$ we have

$$
\begin{gathered}
f\left(\theta+\beta-\frac{x_{1}+y_{1}}{2}\right)=f\left(\frac{\theta+\beta-x_{1}+\theta+\beta-y_{1}}{2}\right) \\
\leq \frac{1}{2}\left[f\left(\theta+\beta-x_{1}\right)+f\left(\theta+\beta-y_{1}\right)\right]
\end{gathered}
$$


for all $x_{1}, y_{1} \in[\theta, \beta]$. With variable replacement $\theta+\beta-x_{1}=\tau(\theta+\beta-x)+$ $(1-\tau)(\theta+\beta-y)$ and $\theta+\beta-y_{1}=(1-\tau)(\theta+\beta-x)+\tau(\theta+\beta-y), x, y \in[\theta, \beta]$ and $\tau \in[0,1]$ in $(2.10)$,we obtain

$$
\begin{aligned}
& f\left(\theta+\beta-\frac{x+y}{2}\right) \\
& \leq \frac{1}{2}[f(\tau(\theta+\beta-x)+(1-\tau)(\theta+\beta-y))+f((1-\tau)(\theta+\beta-x)+\tau(\theta+\beta-y))] .
\end{aligned}
$$

Multiplying both sides of $(2.11)$ by $\frac{\tau^{\sigma-1} e^{\frac{\xi-1}{\xi} \tau}}{\xi^{\sigma}}$ and integrate with respect to $\tau$ over $[0,1]$, we have,

$$
\begin{aligned}
& \frac{1}{\xi^{\sigma}}\left[\frac{1}{\sigma}+\sum_{n=1}^{\infty}\left(\frac{\xi-1}{\xi}\right)^{n} \frac{1}{\sigma+n}\right] f\left(\theta+\beta-\frac{x+y}{2}\right) \\
& \leq \frac{1}{2} \int_{0}^{1} \frac{\tau^{\sigma-1} e^{\frac{\xi-1}{\xi} \tau}}{\xi^{\sigma}} f(\tau(\theta+\beta-x)+(1-\tau)(\theta+\beta-y)) d \tau \\
& +\frac{1}{2} \int_{0}^{1} \frac{\tau^{\sigma-1} e^{\frac{\xi-1}{\xi} \tau}}{\xi^{\sigma}} f((1-\tau)(\theta+\beta-x)+\tau(\theta+\beta-y)) d \tau \\
& =\frac{1}{2 \xi^{\sigma}}\left[\int_{\theta+\beta-y}^{\theta+\beta-x} e^{\frac{\xi-1}{\xi}\left(u-\frac{\theta+\beta-y}{y-x}\right)}\left(u-\frac{\theta+\beta-y}{y-x}\right)^{\sigma-1} f(u) d u\right] \\
& +\frac{1}{2 \xi^{\sigma}}\left[\int_{\theta+\beta-y}^{\theta+\beta-x} e^{\frac{\xi-1}{\xi}\left(u-\frac{\theta+\beta-x}{y-x}\right)}\left(u-\frac{\theta+\beta-x}{y-x}\right)^{\sigma-1} f(u) d u\right] \\
& =\frac{\Gamma(\sigma)}{2(y-x)^{\sigma}}\left[J_{(\theta+\beta-y)^{+}}^{\sigma, \xi} f(\theta+\beta-x)+J_{(\theta+\beta-x)^{-}}^{\sigma, \xi} f(\theta+\beta-y)\right]
\end{aligned}
$$

The proof of first inequality of (2.2) is completed. On the other hand, using the convexity of $f$ we can write

$$
f(\tau(\theta+\beta-x)+(1-\tau)(\theta+\beta-y)) \leq \tau f((\theta+\beta-x))+(1-\tau) f(\theta+\beta-y)
$$

and

$$
f((1-\tau)(\theta+\beta-x)+\tau(\theta+\beta-y)) \leq(1-\tau) f((\theta+\beta-x))+\tau f(\theta+\beta-y)
$$

by adding these inequalities an dusing the Jensen -Mercer inequality, we have

$$
\begin{gathered}
f(\tau(\theta+\beta-x)+(1-\tau)(\theta+\beta-y))+f((1-\tau)(\theta+\beta-x)+\tau(\theta+\beta-y)) \\
\leq f((\theta+\beta-x))+f(\theta+\beta-y) \\
\leq 2[f(\theta)+f(\beta)]-[f(x)+f(y)] .
\end{gathered}
$$

Multiplying both sides of (2.15) by $\frac{\tau^{\sigma-1} e^{\frac{\xi-1}{\xi} \tau}}{\xi^{\sigma}}$ and then integrating the resulting inequality with respect to $\tau$ over $[0,1]$, we have second and third inequalities of $(2.2)$.

Remark 1. If we consider $\xi=1, \sigma=1$ in Theorem, the following inequality is obtain

$$
\begin{aligned}
f\left(\theta+\beta-\frac{x+y}{2}\right) & \leq f(\theta)+f(\beta)-\int_{0}^{1} f(\tau x+(1-\tau) y) d \tau \\
& \leq f(\theta)+f(\beta)-f\left(\frac{x+y}{2}\right)
\end{aligned}
$$

and

$$
\begin{aligned}
f\left(\theta+\beta-\frac{x+y}{2}\right) & \leq \frac{1}{y-x} \int_{x}^{y} f(\theta+\beta-\tau) d \tau \\
& \leq f(\theta)+f(\beta)-\frac{f(x)+f(y)}{2}
\end{aligned}
$$

which is proved by Kian and Moslehian in [18]. 
Theorem 3. Let $f$ be a positive continuous, decreasing and convex function on the interval $[\theta, \beta]$ then the following inequality for, generalized proportional fractional integrals holds;

$$
\begin{aligned}
& \frac{2}{\xi^{\sigma}}\left[\frac{1}{\sigma}+\sum_{n=1}^{\infty}\left(\frac{\xi-1}{\xi}\right)^{n} \frac{1}{\sigma+n}\right] f\left(\theta+\beta-\frac{x+y}{2}\right) \\
& \leq \frac{2^{\sigma} \Gamma(\sigma)}{(y-x)^{\sigma}}\left[J_{\left(\theta+\beta-\frac{x+y}{2}\right.}^{\sigma, \xi} f(\theta+\beta-y)+J_{\left(\theta+\beta-\frac{x+y}{2}\right)^{+}}^{\sigma, \xi}(\theta+\beta-x)\right] \\
& \leq \frac{2}{\xi^{\sigma}}\left[\frac{1}{\sigma}+\sum_{n=1}^{\infty}\left(\frac{\xi-1}{\xi}\right)^{n} \frac{1}{\sigma+n}\right]\left[[f(\theta)+f(\beta)]-\frac{f(x)+f(y)}{2}\right] .
\end{aligned}
$$

Then where $\xi \in(0,1]$ for all $x, y \in[\theta, \beta], \sigma \in \mathbb{C}$ and $\Re(\sigma)>0$.

Proof. To prove the first (2.16), by writting $x_{1}=\frac{\tau}{2} x+\frac{2-\tau}{2} y$ and $y_{1}=\frac{2-\tau}{2} x+\frac{\tau}{2} y$ for $x, y \in[\theta, \beta]$ and $\tau \in[0,1]$ in the inequality $(2.10)$ we get

$$
\begin{gathered}
2 f\left(\theta+\beta-\frac{x+y}{2}\right) \\
\leq\left[f\left(\theta+\beta-\left(\frac{\tau}{2} x+\frac{2-\tau}{2} y\right)\right)+f\left(\theta+\beta-\left(\frac{2-\tau}{2} x+\frac{\tau}{2} y\right)\right)\right]
\end{gathered}
$$

and then, Multiplying both sides of $(2.17)$ by $\frac{\tau^{\sigma-1} e^{\frac{\xi-1}{\xi} \tau}}{\xi^{\sigma}}$ and integrate with respect to $\tau$ over $[0,1]$, we get

$$
\begin{aligned}
& \frac{2}{\xi^{\sigma}}\left[\frac{1}{\sigma}+\sum_{n=1}^{\infty}\left(\frac{\xi-1}{\xi}\right)^{n} \frac{1}{\sigma+n}\right] f\left(\theta+\beta-\frac{x+y}{2}\right) \\
& \leq \int_{0}^{1} \frac{\tau^{\sigma-1} e^{\frac{\xi-1}{\xi} \tau}}{\xi^{\sigma}} f\left(\theta+\beta-\left(\frac{\tau}{2} x+\frac{2-\tau}{2} y\right)\right) d \tau \\
& +\int_{0}^{1} \frac{\tau^{\sigma-1} e^{\frac{\xi-1}{\xi} \tau}}{\xi^{\sigma}} f\left(\theta+\beta-\left(\frac{2-\tau}{2} x+\frac{\tau}{2} y\right)\right) d \tau \\
& =\int_{\theta+\beta-\frac{x+y}{2}}^{2}\left(\frac{2(u-(\theta+\beta-y))}{y-x}\right)^{\sigma-1} e^{\frac{\xi-1}{\xi} \frac{2(u-(\theta+\beta-y))}{y-x}} f(u) \frac{2 d u}{y-x} \\
& +\int_{\theta+\beta-y}^{\theta+\beta-x}\left(\frac{2((\theta+\beta-x)-u)}{y-x}\right)^{\sigma-1} e^{\frac{\xi-1}{\xi} \frac{2((\theta+\beta-x)-u)}{y-x}} f(u) \frac{2 d u}{y-x} \\
& =\frac{2^{\sigma} \Gamma(\sigma)}{(y-x)^{\sigma}}\left[J_{\left(\theta+\beta-\frac{x+y}{2}\right)^{-}}^{\sigma, \xi}(\theta+\beta-y)+J_{\left(\theta+\beta-\frac{x+y}{2}\right.}^{\sigma, \xi}\right.
\end{aligned}
$$

the first inequality $(2.16)$ is proved. To be able to prove the second inequality of (2.16) by using Jensen Mercer inequality, we get

$$
f\left(\theta+\beta-\left(\frac{\tau}{2} x+\frac{2-\tau}{2} y\right)\right) \leq f(\theta)+f(\beta)-\left[\frac{\tau}{2} f(x)+\frac{2-\tau}{2} f(y)\right]
$$

and

$$
f\left(\theta+\beta-\left(\frac{2-\tau}{2} x+\frac{\tau}{2} y\right)\right) \leq f(\theta)+f(\beta)-\left[\frac{2-\tau}{2} f(x)+\frac{\tau}{2} f(y)\right]
$$

by adding these inequalities, we have

$$
\begin{aligned}
& f\left(\theta+\beta-\left(\frac{\tau}{2} x+\frac{2-\tau}{2} y\right)\right)+f\left(\theta+\beta-\left(\frac{2-\tau}{2} x+\frac{\tau}{2} y\right)\right) \\
& \leq 2[f(\theta)+f(\beta)]-\frac{f(x)+f(y)}{2}
\end{aligned}
$$

Multiplying both sides of (2.21) by $\frac{\tau^{\sigma-1} e^{\frac{\xi-1}{\xi} \tau}}{\xi^{\sigma}}$ and integrate with respect to $\tau$ over $[0,1]$, we find second inequality of $(2.16)$. 


\section{Hermite-Hadamard Mercer Type Inequalities For Fractional INTEGRALS}

Now, we give the new following lemmas for our results.

Lemma 1. Let $f$ be a positive continuous, decreasing and convex function a differentiable mapping on $(\theta, \beta)$ with $\theta<\beta$. If $f^{\prime} \in L[\theta, \beta]$, then the following equality for, generalized proportional fractional integrals holds;

$$
\begin{aligned}
& \frac{e^{\frac{\xi-1}{\xi}}}{2}[f(\theta+\beta-x)+f(\theta+\beta-y)] \\
& -\frac{\xi^{\sigma-1}(\xi-1) \Gamma(\sigma)}{2(y-x)^{\sigma+1}}\left[J_{(\theta+\beta-x)^{-}}^{\sigma-1, \xi} f(\theta+\beta-y)+J_{(\theta+\beta-y)^{+}}^{\sigma-1, \xi} f(\theta+\beta-x)\right] \\
& -\frac{\xi^{\sigma} \sigma \Gamma(\sigma)}{2(y-x)^{\sigma}}\left[J_{(\theta+\beta-x)^{-}}^{\sigma, \xi}(\theta+\beta-y)+J_{(\theta+\beta-y)^{+}}^{\sigma, \xi} f(\theta+\beta-x)\right] \\
& =\frac{y-x}{2} \int_{0}^{1} e^{\frac{\xi-1}{\xi} \tau} \tau^{\sigma} f^{\prime}(\theta+\beta-(\tau x+(1-\tau) y)) d \tau \\
& -\frac{y-x}{2} \int_{0}^{1} e^{\frac{\xi-1}{\xi}(1-\tau)}(1-\tau)^{\sigma} f^{\prime}(\theta+\beta-(\tau x+(1-\tau) y)) d \tau
\end{aligned}
$$

Then where $\tau \in[0,1], \xi \in(0,1]$ for all $x, y \in[\theta, \beta], \sigma \in \mathbb{C}$ and $\Re(\sigma)>0$.

Proof. It is necessary to note,

$$
\begin{aligned}
& \int_{0}^{1} e^{\frac{\xi-1}{\xi} \tau} \tau^{\sigma} f^{\prime}(\theta+\beta-(\tau x+(1-\tau) y)) d \tau \\
& -\int_{0}^{1} e^{\frac{\xi-1}{\xi}(1-\tau)}(1-\tau)^{\sigma} f^{\prime}(\theta+\beta-(\tau x+(1-\tau) y)) d \tau \\
& =I_{1}-I_{2}
\end{aligned}
$$

integrating by parts, we get

$$
\begin{aligned}
& I_{1}=\int_{0}^{1} e^{\frac{\xi-1}{\xi} \tau} \tau^{\sigma} f^{\prime}(\theta+\beta-(\tau x+(1-\tau) y)) d \tau \\
& =\left.\frac{e^{\frac{\xi-1}{\xi} \tau} \tau^{\sigma} f(\theta+\beta-(\tau x+(1-\tau) y))}{(y-x)}\right|_{0} ^{1} \\
& -\frac{1}{(y-x)} \int_{0}^{1} e^{\frac{\xi-1}{\xi} \tau}\left(\frac{\xi-1}{\xi} \tau^{\sigma}+\sigma \tau^{\sigma-1}\right) f(\theta+\beta-(\tau x+(1-\tau) y)) d \tau \\
& =\left.\frac{e^{\frac{\xi-1}{\xi} \tau} \tau^{\sigma} f(\theta+\beta-(\tau x+(1-\tau) y))}{(y-x)}\right|_{0} ^{1} \\
& -\frac{(\xi-1)}{\xi(y-x)} \int_{0}^{1} e^{\frac{\xi-1}{\xi} \tau} \tau^{\sigma} f(\theta+\beta-(\tau x+(1-\tau) y)) d \tau \\
& -\frac{\sigma}{(y-x)} \int_{0}^{1} e^{\frac{\xi-1}{\xi} \tau} \tau^{\sigma-1} f(\theta+\beta-(\tau x+(1-\tau) y)) d \tau \\
& =\frac{e^{\frac{\xi-1}{\xi}} f(\theta+\beta-x)}{(y-x)}-\frac{\xi^{\sigma-1}(\xi-1)}{\xi^{\sigma}(y-x)} \int_{\theta+\beta-y}^{\theta+\beta-x} e^{\frac{\xi-1}{\xi}\left(\frac{u-(\theta+\beta-y)}{y-x}\right)}\left(\frac{u-(\theta+\beta-y)}{y-x}\right)^{\sigma} f(u) \frac{d u}{y-x} \\
& -\frac{\xi^{\sigma} \sigma}{\xi^{\sigma}(y-x)} \int_{\theta+\beta-\gamma}^{\theta+\beta-x} e^{\frac{\xi-1}{\xi}\left(\frac{u-(\theta+\beta-y)}{y-x}\right)}\left(\frac{u-(\theta+\beta-y)}{y-x}\right)^{\sigma-1} f(u) \frac{d u}{y-x} \\
& =\frac{e^{\frac{\xi-1}{\xi}} f(\theta+\beta-x)}{(y-x)}-\frac{\xi^{\sigma-1}(\xi-1) \Gamma(\sigma)}{(y-x)^{\sigma+2}} J_{(\theta+\beta-x)}^{\sigma-1, \xi} f(\theta+\beta-y)-\frac{\xi^{\sigma} \sigma \Gamma(\sigma)}{(y-x)^{\sigma+1}} J_{(\theta+\beta-x)^{-}}^{\sigma, \xi} f(\theta+\beta-y)
\end{aligned}
$$


Similary we get

(3.4)

$$
\begin{aligned}
& I_{2}=\int_{0}^{1} e^{\frac{\xi-1}{\xi}(1-\tau)}(1-\tau)^{\sigma} f^{\prime}(\theta+\beta-(\tau x+(1-\tau) y)) d \tau \\
& =\left.\frac{e^{\frac{\xi-1}{\xi}(1-\tau)}(1-\tau)^{\sigma} f(\theta+\beta-(\tau x+(1-\tau) y))}{(y-x)}\right|_{0} ^{1} \\
& -\frac{1}{(y-x)} \int_{0}^{1} e^{\frac{\xi-1}{\xi}(1-\tau)}\left(\frac{\xi-1}{\xi}(1-\tau)^{\sigma}+\sigma(1-\tau)^{\sigma-1}\right) f(\theta+\beta-(\tau x+(1-\tau) y)) d \tau \\
& =\left.\frac{e^{\frac{\xi-1}{\xi}(1-\tau)}(1-\tau)^{\sigma} f(\theta+\beta-(\tau x+(1-\tau) y))}{(y-x)}\right|_{0} ^{1} \\
& -\frac{\xi-1}{\xi(y-x)} \int_{0}^{1} e^{\frac{\xi-1}{\xi}(1-\tau)}(1-\tau)^{\sigma} f(\theta+\beta-(\tau x+(1-\tau) y)) d \tau \\
& -\frac{\sigma}{(y-x)} \int_{0}^{1} e^{\frac{\xi-1}{\xi}(1-\tau)}(1-\tau)^{\sigma-1} f(\theta+\beta-(\tau x+(1-\tau) y)) d \tau \\
& =-\frac{e^{\frac{\xi-1}{\xi}} f(\theta+\beta-y)}{(y-x)}+\frac{\xi^{\sigma-1}(\xi-1)}{\xi^{\sigma}(y-x)} \int_{\theta+\beta-y}^{\theta+\beta} e^{\frac{\xi-1}{\xi}\left(1-\frac{u-(\theta+\beta-y)}{y-x}\right)}\left(1-\frac{u-(\theta+\beta-y)}{y-x}\right)^{\sigma} f(u) \frac{d u}{y-x} \\
& +\frac{\sigma \xi^{\sigma}}{\xi^{\sigma}(y-x)} \int_{\theta+\beta-y}^{\theta+\beta-x} e^{\frac{\xi-1}{\xi}\left(1-\frac{u-(\theta+\beta-y)}{y-x}\right)}\left(1-\frac{u-(\theta+\beta-y)}{y-x}\right)^{\sigma-1} f(u) \frac{d u}{y-x} \\
& =-\frac{e^{\frac{\xi-1}{\xi}} f(\theta+\beta-y)}{(y-x)}+\frac{\xi^{\sigma-1}(\xi-1) \Gamma(\sigma)}{(y-x)^{\sigma+2}} J_{(\theta+\beta-y)}^{\sigma-1, \xi} f(\theta+\beta-x)+\frac{\xi^{\sigma} \sigma \Gamma(\sigma)}{(y-x)^{\sigma+1}} J_{(\theta+\beta-y)^{+}}^{\sigma, \xi} f(\theta+\beta-x)
\end{aligned}
$$

We can write

(3.5)

$$
\begin{aligned}
& I=I_{1}-I_{2} \\
& =\frac{\frac{\xi-1}{\xi}}{\xi^{\sigma}(y-x)}[f(\theta+\beta-x)+f(\theta+\beta-y)]-\frac{(\xi-1) \Gamma(\sigma)}{\xi^{\sigma+1}(y-x)^{\sigma+2}}\left[J_{(\theta+\beta-x)^{-}}^{\sigma-1, \xi} f(\theta+\beta-y)+J_{(\theta+\beta-y)^{+}}^{\sigma-1, \xi} f(\theta+\beta-x)\right] \\
& -\frac{\sigma \Gamma(\sigma)}{\xi^{\sigma}(y-x)^{\sigma+1}}\left[J_{(\theta+\beta-x)^{-}}^{\sigma, \xi} f(\theta+\beta-y)+J_{(\theta+\beta-y)^{+}}^{\sigma, \xi} f(\theta+\beta-x)\right]
\end{aligned}
$$

Multiplying the both sides by $\frac{y-x}{2}$, we proof is obtained (3.1).

Corollary 1. If we consider $\xi=1, \sigma=1$ in Lemma 1, then we have the following equality

$$
\begin{aligned}
& \frac{[f(\theta+\beta-x)+f(\theta+\beta-y)]}{2}- \\
& -\frac{1}{y-x}\left[\int_{\theta+\beta-y}^{\theta+\beta-x} f(u) d u\right] \\
& =\frac{y-x}{2} \int_{0}^{1}(2 \tau-1) f^{\prime}(\theta+\beta-(\tau x+(1-\tau) y)) d \tau .
\end{aligned}
$$

which is proved by Sarikaya and Ogülmüs in [27].

Remark 2. If we consider $\xi=1, \sigma=1, x=\theta, y=\beta$ in Lemma 1, in that case the following equality we get,

$$
\begin{aligned}
& \frac{f(\theta)+f(\beta)}{2}\left[\int_{\theta}^{\beta} f(u) d u\right] \\
& \left.-\frac{1}{\beta-\theta}\right] \\
& =\frac{\beta-\theta}{2} \int_{0}^{1}(2 \tau-1) f^{\prime}(\theta+\beta-(\tau \theta+(1-\tau) \beta)) d \tau .
\end{aligned}
$$

which is proved by Dragomir and Agarwal in [9] . 
Remark 3. If we consider $\xi=1$, in Lemma 1 , in that case the following equality we get,

$$
\begin{aligned}
& f(\theta+\beta-x)+f(\theta+\beta-y) \\
& -\frac{\sigma \Gamma(\sigma)}{2(y-x)^{\sigma}}\left[J_{(\theta+\beta-x)^{-}}^{\sigma} f(\theta+\beta-y)+J_{(\theta+\beta-y)^{+}}^{\sigma} f(\theta+\beta-x)\right] \\
& =\frac{y-x}{2} \int_{0}^{1} \tau^{\sigma} f^{\prime}(\theta+\beta-(\tau x+(1-\tau) y)) d \tau \\
& -\frac{y-x}{2} \int_{0}^{1}(1-\tau)^{\sigma} f^{\prime}(\theta+\beta-(\tau x+(1-\tau) y)) d \tau
\end{aligned}
$$

which is proved by Sarikaya and Ogülmüs in [27].

Lemma 2. Let $f$ be a positive continuous, decreasing and convex function a differentiable mapping on $(\theta, \beta)$ with $\theta<\beta$. If $f^{\prime} \in L[\theta, \beta]$, then the following equality for fractional integrals holds;

$$
\begin{aligned}
& \left.\frac{\xi^{\sigma-1} 2^{\sigma}(\xi-1)}{(y-x)^{\sigma}}\left[J_{\left(\theta+\beta-\frac{x+y}{2}\right.}^{\sigma-1, \xi}\right)^{+} f(\theta+\beta-x)+J_{\left(\theta+\beta-\frac{x+y}{2}\right)^{-}}^{\sigma-1, \xi} f(\theta+\beta-y)\right] \\
& +\frac{\xi^{\sigma} 2^{\sigma-1}}{(y-x)^{\sigma}}\left[J_{\left(\theta+\beta-\frac{x+y}{2}\right)^{+}}^{\sigma, \xi} f(\theta+\beta-x)+J_{\left(\theta+\beta-\frac{x+y}{2}\right)^{-}}^{\sigma, \xi} f(\theta+\beta-y)\right] \\
& -e^{\frac{\xi-1}{\xi}} f\left(\theta+\beta-\frac{x+y}{2}\right) \\
& =\frac{y-x}{4} \int_{0}^{1} e^{\frac{\xi-1}{\xi} \tau} \tau^{\sigma}\left[f^{\prime}\left(\theta+\beta-\left(\frac{2-\tau}{2} x+\frac{\tau}{2} y\right)\right)-f^{\prime}\left(\theta+\beta-\left(\frac{\tau}{2} x+\frac{2-\tau}{2} y\right)\right)\right] d \tau .
\end{aligned}
$$

Then where $\tau \in[0,1], \xi \in(0,1]$ for all $x, y \in[\theta, \beta], \sigma \in \mathbb{C}$ and $\Re(\sigma)>0$.

Proof. It suffices to note that

$$
\begin{aligned}
& \int_{0}^{1} e^{\frac{\xi-1}{\xi} \tau} \tau^{\sigma}\left[f^{\prime}\left(\theta+\beta-\left(\frac{2-\tau}{2} x+\frac{\tau}{2} y\right)\right)-f^{\prime}\left(\theta+\beta-\left(\frac{\tau}{2} x+\frac{2-\tau}{2} y\right)\right)\right] d \tau \\
& =\int_{0}^{1} e^{\frac{\xi-1}{\xi} \tau} \tau^{\sigma} f^{\prime}\left(\theta+\beta-\left(\frac{2-\tau}{2} x+\frac{\tau}{2} y\right)\right) d \tau \\
& -\int_{0}^{1} e^{\frac{\xi-1}{\xi} \tau} \tau^{\sigma} f^{\prime}\left(\theta+\beta-\left(\frac{\tau}{2} x+\frac{2-\tau}{2} y\right)\right) d \tau \\
& =I_{1}-I_{2}
\end{aligned}
$$


Integrating by parts, we get

$$
\begin{aligned}
& I_{1}=\int_{0}^{1} e^{\frac{\xi-1}{\xi} \tau} \tau^{\sigma} f^{\prime}\left(\theta+\beta-\left(\frac{2-\tau}{2} x+\frac{\tau}{2} y\right)\right) d \tau \\
& =\left.e^{\frac{\xi-1}{\xi} \tau} \tau^{\sigma} \frac{2}{y-x} f\left(\theta+\beta-\left(\frac{2-\tau}{2} x+\frac{\tau}{2} y\right)\right)\right|_{0} ^{1} \\
& -\int_{0}^{1} \frac{2}{y-x} e^{\frac{\xi-1}{\xi} \tau}\left[\frac{\xi-1}{\xi} \tau^{\sigma}+\sigma \tau^{\sigma-1}\right] f\left(\theta+\beta-\left(\frac{2-\tau}{2} x+\frac{\tau}{2} y\right)\right) \\
& =e^{\frac{\xi-1}{\xi}} \frac{2}{y-x} f\left(\theta+\beta-\frac{x+y}{2}\right) \\
& -\frac{2(\xi-1)}{\xi(y-x)} \int_{0}^{1} e^{\frac{\xi-1}{\xi} \tau} \tau^{\sigma} f\left(\theta+\beta-\left(\frac{2-\tau}{2} x+\frac{\tau}{2} y\right)\right) d \tau \\
& -\frac{2 \sigma}{(y-x)} \int_{0}^{1} e^{\frac{\xi-1}{\xi} \tau} \tau^{\sigma-1} f\left(\theta+\beta-\left(\frac{2-\tau}{2} x+\frac{\tau}{2} y\right)\right) \\
& =e^{\frac{\xi-1}{\xi}} \frac{2}{y-x} f\left(\theta+\beta-\frac{x+y}{2}\right) \\
& -\frac{2(\xi-1)}{\xi(y-x)} \int_{\theta+\beta-\frac{x+y}{2}}^{\theta+\beta-x} e^{\frac{\xi-1}{\xi}\left[\frac{2((\theta+\beta-x)-u)}{y-x}\right]}\left(\frac{2((\theta+\beta-x)-u)}{y-x}\right)^{\sigma} f(u)\left(\frac{-2 d u}{y-x}\right) \\
& -\frac{2 \sigma}{(y-x)} \int_{\theta+\beta-x} e^{\frac{\xi-1}{\xi}\left[\frac{2((\theta+\beta-x)-u)}{y-x}\right]}\left(\frac{2((\theta+\beta-x)-u)}{y-x}\right)^{\sigma-1} f(u)\left(\frac{-2 d u}{y-x}\right) \\
& =-e^{\frac{\xi-1}{\xi}} \frac{2}{y-x} f\left(\theta+\beta-\frac{x+y}{2}\right) \\
& \left.+\frac{\xi^{\sigma-1} 2^{\sigma+2}(\xi-1)}{(y-x)^{\sigma+1}} J_{(\theta-1, \xi}^{\sigma-\frac{x+y}{2}}\right)^{+} f(\theta+\beta-x) \\
& +\frac{\xi^{\sigma} 2^{\sigma+1}}{(y-x)^{\sigma+1}} J_{\left(\theta+\beta-\frac{x+y}{2}\right.}^{\sigma, \xi} f(\theta+\beta-x) .
\end{aligned}
$$

Similary we get

$$
\begin{aligned}
& I_{2}=\int_{0}^{1} e^{\frac{\xi-1}{\xi} \tau} \tau^{\sigma} f^{\prime}\left(\theta+\beta-\left(\frac{\tau}{2} x+\frac{2-\tau}{2} y\right)\right) d \tau \\
& =\left.e^{\frac{\xi-1}{\xi} \tau} \tau^{\sigma} \frac{2}{y-x} f\left(\theta+\beta-\left(\frac{\tau}{2} x+\frac{2-\tau}{2} y\right)\right)\right|_{0} ^{1} \\
& -\int_{0}^{1} \frac{2}{y-x} e^{\frac{\xi-1}{\xi} \tau}\left[\frac{\xi-1}{\xi} \tau^{\sigma}+\sigma \tau^{\sigma-1}\right] f\left(\theta+\beta-\left(\frac{\tau}{2} x+\frac{2-\tau}{2} y\right)\right) \\
& =e^{\frac{\xi-1}{\xi}} \frac{2}{y-x} f\left(\theta+\beta-\frac{x+y}{2}\right) \\
& -\frac{2(\xi-1)}{\xi(y-x)} \int_{0}^{1} e^{\frac{\xi-1}{\xi} \tau} \tau^{\sigma} f\left(\theta+\beta-\left(\frac{\tau}{2} x+\frac{2-\tau}{2} y\right)\right) d \tau \\
& -\frac{2 \sigma}{(y-x)} \int_{0}^{1} e^{\frac{\xi-1}{\xi} \tau} \tau^{\sigma-1} f\left(\theta+\beta-\left(\frac{\tau}{2} x+\frac{2-\tau}{2} y\right)\right) \\
& =e^{\frac{\xi-1}{\xi}} \frac{2}{y-x} f\left(\theta+\beta-\frac{x+y}{2}\right) \\
& -\frac{2(\xi-1)}{\xi(y-x)} \int_{\theta+\beta-\frac{x+y}{2}} e^{\frac{\xi-1}{\xi}\left[\frac{2(u-(\theta+\beta-y))}{y-x}\right]}\left(\frac{2(u-(\theta+\beta-y))}{y-x}\right)^{\sigma} f(u)\left(\frac{2 d u}{y-x}\right) \\
& -\frac{2 \sigma}{(y-x)} \int_{\theta+\beta-\frac{x+y}{2}} e^{\frac{\xi-1}{\xi}\left[\frac{2(u-(\theta+\beta-y))}{y-x}\right]}\left(\frac{2(u-(\theta+\beta-y))}{y-x}\right)^{\sigma-1} f(u)\left(\frac{2 d u}{y-x}\right) \\
& =e^{\frac{\xi-1}{\xi}} \frac{2}{y-x} f\left(\theta+\beta-\frac{x+y}{2}\right) \\
& -\frac{\xi^{\sigma-1} 2^{\sigma-2}(\xi-1)}{(y-x)^{\sigma+1}} J_{(\theta-1, \xi}^{\left.\sigma-\frac{x+y}{2}\right)^{-}} f(\theta+\beta-y) \\
& -\frac{\xi^{\sigma} 2^{\sigma+1}}{(y-x)^{\sigma+1}} J_{\left(\theta+\beta-\frac{x+y}{2}\right)^{-}}^{\sigma(\theta)}(\theta+\beta-y) .
\end{aligned}
$$


We can write

(3.11)

$$
\begin{aligned}
& I_{1}-I_{2}=-e^{\frac{\xi-1}{\xi}} \frac{4}{y-x} f\left(\theta+\beta-\frac{x+y}{2}\right) \\
& +\frac{\xi^{\sigma-1} 2^{\sigma+2}(\xi-1)}{(y-x)^{\sigma+1}}\left[J_{\left(\theta+\beta-\frac{x+y}{2}\right)^{+}}^{\sigma-1, \xi} f(\theta+\beta-x)+J_{\left(\theta+\beta-\frac{x+y}{2}\right.}^{\sigma-1, \xi}\right. \\
& +\frac{\xi^{\sigma} 2^{\sigma+1}}{(y-x)^{\sigma+1}}\left[J_{\left(\theta+\beta-\frac{x+y}{2}\right)^{+}}^{\sigma, \xi} f(\theta+\beta-x)+J_{\left(\theta+\beta-\frac{x+y}{2}\right)^{-}}^{\sigma, \xi}(\theta+\beta-y)\right] .
\end{aligned}
$$

Multiplying the both sides by $\frac{y-x}{4}$, we obtain the conclusion (3.6).

Remark 4. If we consider $x=\theta, y=\beta$ and $\xi=1$ in Theorem 2 becomes Theorem 3 proved by Sarikaya et. al in [25].

Remark 5. If we consider $x=\theta, \sigma=1, y=\beta$ and $\xi=1$ in Theorem 2 becomes Theorem 2 gives [9, Theorem2.2] .

Remark 6. If we consider $\xi=1$, in Lemma 2, in that case the following equality we get,

$$
\begin{aligned}
& \frac{2^{\sigma-1}}{(y-x)^{\sigma}}\left[J_{\left(\theta+\beta-\frac{x+y}{2}\right)^{+}}^{\sigma} f(\theta+\beta-x)+J_{\left(\theta+\beta-\frac{x+y}{2}\right)^{-}}^{\sigma} f(\theta+\beta-y)\right] \\
& -f\left(\theta+\beta-\frac{x+y}{2}\right) \\
& =\frac{y-x}{4} \int_{0}^{1} \tau^{\sigma}\left[f^{\prime}\left(\theta+\beta-\left(\frac{2-\tau}{2} x+\frac{\tau}{2} y\right)\right)-f^{\prime}\left(\theta+\beta-\left(\frac{\tau}{2} x+\frac{2-\tau}{2} y\right)\right)\right] d \tau
\end{aligned}
$$

which is proved by Sarikaya and Ogülmüş in [27].

Theorem 4. Let $f$ be a positive continuous, decreasing and convex function a differentiable mapping on $(\theta, \beta)$ with $\theta<\beta$. If $\left|f^{\prime}\right|$ is convex on $[\theta, \beta]$, then the following inequality for fractional integrals holds;

$$
\begin{aligned}
& \mid \frac{e^{\frac{\xi-1}{\xi}}}{2}[f(\theta+\beta-x)+f(\theta+\beta-y)] \\
& -\frac{\xi^{\sigma-1}(\xi-1) \Gamma(\sigma)}{2(y-x)^{\sigma+1}}\left[J_{(\theta+\beta-x)^{-}}^{\sigma-1, \xi} f(\theta+\beta-y)+J_{(\theta+\beta-y)^{+}}^{\sigma-1, \xi} f(\theta+\beta-x)\right] \\
& -\frac{\xi^{\sigma} \sigma \Gamma(\sigma)}{2(y-x)^{\sigma}}\left[J_{(\theta+\beta-x)^{-}}^{\sigma, \xi}(\theta+\beta-y)+J_{(\theta+\beta-y)^{+}}^{\sigma, \xi} f(\theta+\beta-x)\right] \mid \\
& \leq\left(\left|f^{\prime}(\theta)\right|+\left|f^{\prime}(\beta)\right|-\frac{\left|f^{\prime}(x)\right|+\left|f^{\prime}(y)\right|}{2}\right) \\
& \times\left(\frac{1}{\sigma+1}-\frac{\frac{1}{2 \sigma}}{\sigma+1}+\sum_{n=1}^{\infty}\left(\frac{\xi-1}{\xi}\right)^{n} \frac{1}{n !}\left(\frac{1}{\sigma+n+1}-\frac{\frac{1}{2 \sigma+n}}{\sigma+1+n}\right)\right)
\end{aligned}
$$

Then where $\tau \in[0,1], \xi \in(0,1]$ for all $x, y \in[\theta, \beta], \sigma \in \mathbb{C}$ and $\Re(\sigma)>0$. 
HERMITE-HADAMARD- MERCER TYPE INEQUALITIES...

Proof. By means of the Lemma 1 and Jensen Mercur inequality, we find that (3.13)

$$
\begin{aligned}
& \mid \frac{e^{\frac{\xi-1}{\xi}}}{2}[f(\theta+\beta-x)+f(\theta+\beta-y)]-\frac{\xi^{\sigma-1}(\xi-1) \Gamma(\sigma)}{2(y-x)^{\sigma+1}}\left[J_{(\theta+\beta-x)^{-}}^{\sigma-1, \xi} f(\theta+\beta-y)+J_{(\theta+\beta-y)^{+}}^{\sigma-1, \xi} f(\theta+\beta-x)\right] \\
& -\frac{\xi^{\sigma} \sigma \Gamma(\sigma)}{2(y-x)^{\sigma}}\left[J_{(\theta+\beta-x)^{-}}^{\sigma, \xi} f(\theta+\beta-y)+J_{(\theta+\beta-y)^{+}}^{\sigma, \xi} f(\theta+\beta-x)\right] \mid \\
& \leq \frac{y-x}{2} \int_{0}^{1}\left|e^{\frac{\xi-1}{\xi} \tau} \tau^{\sigma}-e^{\frac{\xi-1}{\xi}(1-\tau)}(1-\tau)^{\sigma}\right|\left|f^{\prime}(\theta+\beta-(\tau x+(1-\tau) y))\right| d \tau \\
& =\frac{y-x}{2}\left\{\int_{0}^{\frac{1}{2}}\left[e^{\frac{\xi-1}{\xi}(1-\tau)}(1-\tau)^{\sigma}-e^{\frac{\xi-1}{\xi} \tau} \tau^{\sigma}\right]\left[\left|f^{\prime}(\theta)\right|+\left|f^{\prime}(\beta)\right|-\left(\tau\left|f^{\prime}(x)\right|+(1-\tau)\left|f^{\prime}(y)\right|\right)\right]\right. \\
& \left.\int_{\frac{1}{2}}^{1}\left[e^{\frac{\xi-1}{\xi} \tau} \tau^{\sigma}-e^{\frac{\xi-1}{\xi}(1-\tau)}(1-\tau)^{\sigma}\right]\left[\left|f^{\prime}(\theta)\right|+\left|f^{\prime}(\beta)\right|-\left(\tau\left|f^{\prime}(x)\right|+(1-\tau)\left|f^{\prime}(y)\right|\right)\right]\right\} \\
& =\frac{y-x}{2}\left(R_{1}+R_{2}\right) .
\end{aligned}
$$

calculating $R_{1}$ and $R_{2}$, we obtain

$$
\begin{aligned}
& R_{1}=\left(\left|f^{\prime}(\theta)\right|+\left|f^{\prime}(\beta)\right|\right)\left(\int_{0}^{\frac{1}{2}}\left(e^{\frac{\xi-1}{\xi}(1-\tau)}(1-\tau)^{\sigma}-e^{\frac{\xi-1}{\xi} \tau} \tau^{\sigma}\right) d \tau\right) \\
& -\left|f^{\prime}(x)\right|\left[\int_{0}^{\frac{1}{2}} e^{\frac{\xi-1}{\xi}(1-\tau)}(1-\tau)^{\sigma} \tau d \tau-\int_{0}^{\frac{1}{2}} e^{\frac{\xi-1}{\xi} \tau} \tau^{\sigma+1} d \tau\right] \\
& +\left|f^{\prime}(y)\right|\left[\int_{0}^{\frac{1}{2}} e^{\frac{\xi-1}{\xi}(1-\tau)}(1-\tau)^{\sigma+1} d \tau-\int_{0}^{\frac{1}{2}} e^{\frac{\xi-1}{\xi} \tau} \tau^{\sigma}(1-\tau) d \tau\right] \\
& =\left(\left|f^{\prime}(\theta)\right|+\left|f^{\prime}(\beta)\right|\right)\left(\frac{1}{\sigma+1}-\frac{\frac{1}{2^{\sigma}}}{\sigma+1}+\sum_{n=1}^{\infty} \frac{\left(\frac{\xi-1}{\xi}\right)^{n}\left(\frac{1}{2}\right)^{\sigma+1+n} 2}{(\sigma+n+1) n !}\right) \\
& -\left\{\left|f^{\prime}(x)\right|\left(\frac{1}{(\sigma+1)(\sigma+2)}-\frac{1}{\sigma+1}+\sum_{n=1}^{\infty}\left(\frac{\xi-1}{\xi}\right)^{n} \frac{1}{n !}\left[\frac{1}{(\sigma+1+n)(\sigma+2+n)}-\frac{\frac{1}{2^{\sigma+n+1}}}{(\sigma+1+n)}\right]\right)\right. \\
& \left.+\left|f^{\prime}(y)\right|\left(\frac{1}{\sigma+2}-\frac{1}{\sigma+1}+\sum_{n=1}^{\infty}\left(\frac{\xi-1}{\xi}\right)^{n} \frac{1}{n !}\left[\frac{1}{(\sigma+2+n)}-\frac{\frac{1}{2^{\sigma+n+1}}}{(\sigma+1+n)}\right]\right)\right\}
\end{aligned}
$$

and

$$
\begin{aligned}
& R_{2}=\left(\left|f^{\prime}(\theta)\right|+\left|f^{\prime}(\beta)\right|\right)\left(\int_{\frac{1}{2}}^{1}\left(e^{\frac{\xi-1}{\xi} \tau} \tau^{\sigma}-e^{\frac{\xi-1}{\xi}(1-\tau)}(1-\tau)^{\sigma}\right) d \tau\right) \\
& -\left|f^{\prime}(x)\right|\left[\int_{\frac{1}{2}}^{1} e^{\frac{\xi-1}{\xi} \tau} \tau^{\sigma+1} d \tau-\int_{\frac{1}{2}}^{1} e^{\frac{\xi-1}{\xi}(1-\tau)}(1-\tau)^{\sigma} \tau d \tau\right] \\
& +\left|f^{\prime}(y)\right|\left[\int_{\frac{1}{2}}^{1} e^{\frac{\xi-1}{\xi} \tau} \tau^{\sigma}(1-\tau) d \tau-\int_{\frac{1}{2}}^{1} e^{\frac{\xi-1}{\xi}(1-\tau)}(1-\tau)^{\sigma+1} d \tau\right] \\
& =\left(\left|f^{\prime}(\theta)\right|+\left|f^{\prime}(\beta)\right|\right)\left(\frac{1}{\sigma+1}-\frac{\frac{1}{2^{\sigma}}}{\sigma+1}+\sum_{n=1}^{\infty} \frac{\left(\frac{\xi-1}{\xi}\right)^{n}\left(\frac{1}{2}\right)^{\sigma+1+n} 2}{(\sigma+n+1) n !}\right) \\
& -\left\{\left|f^{\prime}(x)\right|\left(\frac{1}{\sigma+2}-\frac{\frac{1}{2^{\sigma+1}}}{\sigma+1}+\sum_{n=1}^{\infty}\left(\frac{\xi-1}{\xi}\right)^{n} \frac{1}{n !}\left[\frac{1}{(\sigma+2+n)}-\frac{\frac{1}{2^{\sigma+n+1}}}{(\sigma+1+n)}\right]\right)\right. \\
& \left.+\left|f^{\prime}(y)\right|\left(\frac{1}{(\sigma+1)(\sigma+2)}-\frac{\frac{1}{2^{\sigma+1}}}{\sigma+1}+\sum_{n=1}^{\infty}\left(\frac{\xi-1}{\xi}\right)^{n} \frac{1}{n !}\left[\frac{1}{(\sigma+1+n)(\sigma+2+n)}-\frac{\frac{1}{2^{\sigma+n+1}}}{(\sigma+1+n)}\right]\right)\right\}
\end{aligned}
$$

By adding $R_{1}$ and $R_{2}$, we get the inequality (3.11). 
Remark 7. If we consider $x=\theta, y=\beta$ and $\xi=1$ in Theorem 2 becomes Theorem 3 proved by Sarikaya et. al in [25].

Remark 8. If we consider $x=\theta, \sigma=1, y=\beta$ and $\xi=1$ in Theorem 2 becomes Theorem 2 gives [9, Theorem2.2].

Remark 9. If we consider $\xi=1$, in Theorem 4 , in that case the following inequality we get,

$$
\begin{aligned}
& \mid f(\theta+\beta-x)+f(\theta+\beta-y) \\
& -\frac{\sigma \Gamma(\sigma)}{2(y-x)^{\sigma}}\left[J_{(\theta+\beta-x)^{-}}^{\sigma} f(\theta+\beta-y)+J_{(\theta+\beta-y)^{+}}^{\sigma} f(\theta+\beta-x)\right] \mid \\
& \leq\left(\left|f^{\prime}(\theta)\right|+\left|f^{\prime}(\beta)\right|-\frac{\left|f^{\prime}(x)\right|+\left|f^{\prime}(y)\right|}{2}\right)
\end{aligned}
$$

which is proved by Sarikaya and Ogülmüş in [27].

Theorem 5. Let $f$ be a positive continuous, decreasing and convex function a differentiable mapping on $(\theta, \beta)$ with $\theta<\beta$. If $\left|f^{\prime}\right|$ is convex on $[\theta, \beta]$, then the following inequality for fractional integrals holds;

$$
\begin{aligned}
& \mid \frac{\xi^{\sigma-1} 2^{\sigma}(\xi-1)}{(y-x)^{\sigma}}\left[J_{\left(\theta+\beta-\frac{x+y}{2}\right)^{+}}^{\sigma-1, \xi} f(\theta+\beta-x)+J_{\left(\theta+\beta-\frac{x+y}{2}\right)^{-}}^{\sigma-1, \xi} f(\theta+\beta-y)\right] \\
& +\frac{\xi^{\sigma} 2^{\sigma-1}}{(y-x)^{\sigma}}\left[J_{\left(\theta+\beta-\frac{x+y}{2}\right)^{\sigma}}^{\sigma, \xi} f(\theta+\beta-x)+J_{\left(\theta+\beta-\frac{x+y}{2}\right)^{-}}^{\sigma, \xi}(\theta+\beta-y)\right] \\
& -e^{\frac{\xi-1}{\xi}} f\left(\theta+\beta-\frac{x+y}{2}\right) \mid \\
& \leq \frac{y-x}{2}\left[\frac{1}{\sigma+1}+\sum_{n=1}^{\infty} \frac{1}{n !}\left(\frac{\xi-1}{\xi}\right)^{n} \frac{1}{\sigma+n+1}\right]\left(\left|f^{\prime}(\theta)\right|+\left|f^{\prime}(\beta)\right|-\frac{\left|f^{\prime}(x)\right|+\left|f^{\prime}(y)\right|}{2}\right)
\end{aligned}
$$

Then where $\tau \in[0,1], \xi \in(0,1]$ for all $x, y \in[\theta, \beta], \sigma \in \mathbb{C}$ and $\Re(\sigma)>0$. 
Proof. Using the Lemma 2 and Jensen -Mercer inequality, we find

$$
\begin{aligned}
& \mid \frac{\xi^{\sigma-1} 2^{\sigma}(\xi-1)}{(y-x)^{\sigma}}\left[J_{\left(\theta+\beta-\frac{x+y}{2}\right)^{+}}^{\sigma-1, \xi}(\theta+\beta-x)+J_{\left(\theta+\beta-\frac{x+y}{2}\right)^{-}}^{\sigma-1, \xi}(\theta+\beta-y)\right] \\
& +\frac{\xi^{\sigma} 2^{\sigma-1}}{(y-x)^{\sigma}}\left[J_{\left(\theta+\beta-\frac{x+y}{2}\right)^{+}}^{\sigma, \xi}(\theta+\beta-x)+J_{\left(\theta+\beta-\frac{x+y}{2}\right)^{-}}^{\sigma, \xi}(\theta+\beta-y)\right] \\
& -e^{\frac{\xi-1}{\xi}} f\left(\theta+\beta-\frac{x+y}{2}\right) \mid \\
& \leq \frac{y-x}{4} \int_{0}^{1} e^{\frac{\xi-1}{\xi} \tau} \tau^{\sigma}\left|f^{\prime}\left(\theta+\beta-\left(\frac{2-\tau}{2} x+\frac{\tau}{2} y\right)\right)\right| d \tau \\
& +\frac{y-x}{4} \int_{0}^{1} e^{\frac{\xi-1}{\xi} \tau} \tau^{\sigma}\left|f^{\prime}\left(\theta+\beta-\left(\frac{\tau}{2} x+\frac{2-\tau}{2} y\right)\right)\right| d \tau \\
& =\frac{y-x}{4} \int_{0}^{1} e^{\frac{\xi-1}{\xi} \tau} \tau^{\sigma}\left[\left|f^{\prime}(\theta)\right|+\left|f^{\prime}(\beta)\right|-\left(\frac{2-\tau}{2}\left|f^{\prime}(x)\right|+\frac{\tau}{2}\left|f^{\prime}(y)\right|\right)\right] \\
& +\frac{y-x}{4} \int_{0}^{1} e^{\frac{\xi-1}{\xi} \tau} \tau^{\sigma}\left[\left|f^{\prime}(\theta)\right|+\left|f^{\prime}(\beta)\right|-\left(\frac{\tau}{2}\left|f^{\prime}(x)\right|+\frac{2-\tau}{2}\left|f^{\prime}(y)\right|\right)\right] \\
& =\frac{y-x}{4}\left(\left|f^{\prime}(\theta)\right|+\left|f^{\prime}(\beta)\right|\right)\left[\frac{1}{\sigma+1}+\sum_{n=1}^{\infty} \frac{1}{n !}\left(\frac{\xi-1}{\xi}\right)^{n} \frac{1}{\sigma+n+1}\right] \\
& -\frac{y-x}{4}\left|f^{\prime}(x)\right|\left[\frac{1}{\sigma+1}-\frac{1}{2(\sigma+2)}+\sum_{n=1}^{\infty}\left(\frac{\xi-1}{\xi}\right)^{n} \frac{1}{n !}\left(\frac{1}{\sigma+n+1}-\frac{1}{2(\sigma+n+2)}\right)\right] \\
& -\frac{y-x}{4}\left|f^{\prime}(y)\right|\left[\frac{1}{2(\sigma+2)}+\sum_{n=1}^{\infty}\left(\frac{\xi-1}{\xi}\right)^{n} \frac{1}{n !}\left(\frac{1}{2(\sigma+n+2)}\right)\right] \\
& +\frac{y-x}{4}\left(\left|f^{\prime}(\theta)\right|+\left|f^{\prime}(\beta)\right|\right)\left[\frac{1}{\sigma+1}+\sum_{n=1}^{\infty} \frac{1}{n !}\left(\frac{\xi-1}{\xi}\right)^{n} \frac{1}{\sigma+n+1}\right] \\
& -\frac{y-x}{4}\left|f^{\prime}(x)\right|\left[\frac{1}{2(\sigma+2)}+\sum_{n=1}^{\infty}\left(\frac{\xi-1}{\xi}\right)^{n} \frac{1}{n !}\left(\frac{1}{2(\sigma+n+2)}\right)\right] \\
& -\frac{y-x}{4}\left|f^{\prime}(y)\right|\left[\frac{1}{\sigma+1}-\frac{1}{2(\sigma+2)}+\sum_{n=1}^{\infty}\left(\frac{\xi-1}{\xi}\right)^{n} \frac{1}{n !}\left(\frac{1}{\sigma+n+1}-\frac{1}{2(\sigma+n+2)}\right)\right]
\end{aligned}
$$

Remark 10. If we consider $\xi=1$, in Theorem 5 , in that case the following inequality we get,

$$
\begin{aligned}
& \left.\mid \frac{2^{\sigma-1}}{(y-x)^{\sigma}}\left[J_{\left(\theta+\beta-\frac{x+y}{2}\right.}^{\sigma}\right)^{+} f(\theta+\beta-x)+J_{\left(\theta+\beta-\frac{x+y}{2}\right)^{-}}^{\sigma} f(\theta+\beta-y)\right] \\
& -f\left(\theta+\beta-\frac{x+y}{2}\right) \mid \\
& \leq \frac{y-x}{2}\left(\left|f^{\prime}(\theta)\right|+\left|f^{\prime}(\beta)\right|-\frac{\left|f^{\prime}(x)\right|+\left|f^{\prime}(y)\right|}{2}\right)
\end{aligned}
$$

which is proved by Sarikaya and Ogülmüs in [27].

Corollary 2. If we consider $\xi=1$ and $\sigma=1$, in Theorem 5, in that case the following inequality we get,

$$
\begin{aligned}
& \left|\frac{1}{y-x} \int_{\theta+\beta-y}^{\theta+\beta-x} f(u) d u-f\left(\theta+\beta-\frac{x+y}{2}\right)\right| \\
& \leq \frac{y-x}{4}\left[\left|f^{\prime}(\theta)\right|+\left|f^{\prime}(\beta)\right|-\frac{\left|f^{\prime}(x)\right|+\left|f^{\prime}(y)\right|}{2}\right]
\end{aligned}
$$

which is proved by Sarikaya and Ogülmüs in [27] . 


\section{REFERENCES}

[1] M. M. Ali and A. R. Khan, Generalized integral Mercer's inequality and integral means, Journal of Inequalities and Special Functions, 10 (1) (2019), pp. 60-76.

[2] T. Ali, M. A. Khan and Y. Khurshidi, Hermite-Hadamard inequality for fractional integrals via eta-convex functions, Acta Mathematica Universitatis Comenianae, 86(1), (2017), 153164.

[3] T. Abdeljawad, On conformable fractional calculus. J. Comput. Appl. Math. 279, 57-66 (2015). https://doi.org/10.1016/j.cam.2014.10.016

[4] B. Ahmad, A. Alsaedi, M. Kirane, B.T. Torebek. Hermite-Hadamard, Hermite-HadamardFejér, Dragomir-Agarwal and Pachpatte type inequalities for convex functions via new fractional integrals. Journal of Computational and Applied Mathematics, (2019) 353, pp. 120-129.

[5] Caputo, M., Fabrizio, M.: A new definition of fractional derivative without singular kernel. Prog. Fract. Differ. Appl. 1(2), 73-85 (2015).

[6] H. Budak, P. Agarwal, New generalized midpoint type inequalities for fractional integral, ResearchGate: https://www.researchgate.net/publication/323199157.

[7] F. Jarad, T. Abdeljawad, J. Alzabut, Generalized fractional derivatives generated by a class of local proportional derivatives. Eur. Phys. J. Spec. Top. 226, 3457-3471 (2017). https://doi.org/10.1140/epjst/e2018-00021-7.

[8] S. S. Dragomir and C. E. M. Pearce, Selected Topics on Hermite-Hadamard Inequalities and Applications, RGMIA Monographs, Victoria University, 2000.

[9] S. S. Dragomir, R. P. Agarwal, Two inequalities for differentiable mappings and applications to special means of real numbers and to trapezoidal formula, Appl. Math. lett. 11 (5) (1998) 91-95.

[10] M.Z. Sarikaya, H. Budak, Generalized Ostrowski type inequalities for local fractional integrals. Proc. Am. Math. Soc. 145(4), 1527-1538 (2017).

[11] E. Set, M.A. Noor, M.U Awan, A. Gözpinar, Generalized Hermite-Hadamard type inequalities involving fractional integral operators. J. Inequal. Appl. 2017, 169 (2017).

[12] F. Ertugral, M. Z. Sarikaya and H. Budak, On refinements of Hermite-Hadamard-Fejér type inequalities for fractional integral operators, Applications and Applied Mathematics, Vol. 13, Issue 1 (June 2018), pp. 426 - 442.

[13] M.Z. Sarikaya, Z Dahmani, M.E. Kiris, F. Ahmad, (k, s)-Riemann-Liouville fractional integral and applications. Hacet. J. Math. Stat. 45(1), 77-89 (2016).

[14] E. Set, M. Tomar, M.Z. Sarikaya, On generalized Grüss type inequalities for k-fractional integrals. Appl. Math. Comput. 269, 29-34 (2015).

[15] R. Gorento, F. Mainardi, Fractional calculus: integral and differential equations of fractional order, Springer Verlag, Wien (1997), 223-276.

[16] Rahman, G., Nisar, K.S., Qi, F.: Some new inequalities of the Gruss type for conformable fractional integrals. AIMS Math. 3(4), 575-583 (2018).

[17] Rahman, G., Khan, A., Abdeljawad, T., Nisar, K.S.: The Minkowski inequalities via generalized proportional fractional integral operators. Adv. Differ. Equ. 2019, 287 (2019). https://doi.org/10.1186/s13662-019-2229-7.

[18] M. Kian and M.S. Moslehian, Refinements of the operator Jensen-Mercer inequality, Electron. J. Linear Algebra, 26:742-753, 2013.

[19] A. A. Kilbas, H. M. Srivastava and J. J. Trujillo, Theory and applications of fractional differential equations, North-Holland Mathematics Studies, 204, Elsevier Sci. B.V., Amsterdam, 2006.

[20] A. Matkovi'c, J. Pecari'c and I. Peri 'c, A variant of Jensenís inequality of Mercerís type for operators with applications. Linear Algebra Appl. 418 (2006), no. 2-3, 551-564.

[21] A. McD. Mercer, A Variant of Jensen's Inequality, J. Ineq. Pure and Appl. Math 4 (4) (2003), Article 73.

[22] D. S. Mitrinovi'c J. E. Pe $\mu$ cari'c and A. M. Fink, Classical and new inequalities in analysis, Kluwer Academic Publishers Group, Dordrecht, 1993.

[23] M. Niezgoda, A generalization of Mercer's result on convex functions, Nonlinear Analysis 71 (2009), 277.

[24] S. G. Samko, A. A. Kilbas, and O. I. Marichev, Fractional Integrals and Derivatives Theory and Application, Gordan and Breach Science, New York, NY, USA, 1993. 
[25] M. Z. Sarikaya, E. Set, H. Yaldiz, and N. Basak, Hermite-Hadamard's inequalities for fractional integrals and related fractional inequalities, Mathematical and Computer Modelling, 57 (2013), pp.2403-2407, https://doi.:10.1016/j.mcm.2011.12.048.

[26] M. Z. Sarikaya and H. Yildirim, On Hermite-Hadamard type inequalities for RiemannLiouville fractional integrals, Miskolc Mathematical Notes, 17 (2016), No. 2, pp. 1049-1059.

[27] H.Ogülmüş and M.Z.Sarikaya, Hermite-Hadamard- Mercer Type Inequalities for Fractional Integrals, December 2019, doi:10.13140/RG.2.2.30669.79844.

[28] E. Set, S. S. Dragomir, A. Gozpinar, Some generalized Hermite-Hadamard type inequalities involving fractional integral operator for functions whose second derivatives in absolute value are s-convex, Res. Rep. Coll., 20 (2017), Art. 14.

[29] G.Rahman, t. Abdeljawad, A. Khan and K.Sooppy Nisar, Some Fractional proportional integral inequalities, Journal of Inequalities and Applications, (2019) 2019:244, https://doi.org/10.1186/s13660-019-2199-z.

[30] G. Rahman, K.S. Nisar, S. Mubeen, J. Choi, Certain inequalities involving the (k, $\rho$ )-fractional integral operator. Far East J. Math. Sci.: FJMS 103(11), 1879-1888 (2018).

[31] T. Abdeljawad, D. Baleanu, Monotonicity results for fractional difference operators with discrete exponential kernels. Adv. Differ. Equ. 2017, 78 (2017). https://doi.org/10.1186/s13662017-1126-1.

[32] T. Abdeljawad, D. Baleanu, On fractional derivatives with exponential kernel and their discrete versions. Rep. Math. Phys. 80, 11-27 (2017). https://doi.org/10.1016/S00344877(17)30059-9.

[33] J. Losada, J. J. Nieto, Properties of a new fractional derivative without singular kernel. Prog. Fract. Differ. Appl. 1(2), 87-92 (2015).

[ Department of Mathematics, Faculty of Science and Arts, University of Kahramanmaraş SÜtçü İmam, 46000, Kahramanmaraş, Turkey

E-mail address: sedakilincmath@gmail.com

E-mail address: hyildir@ksu.edu.tr

[ Department of Mathematics, Faculty of Science and Arts, University of Kahramanmaraş SÜtçÜ İmam, 46000, Kahramanmaraş, Turkey 